\title{
Coupled-Mode Theory derivation of the formal equivalence between a three-mode waveguide and a set of three mutually coupled single-mode waveguides
}

\author{
Yann G. Boucher ${ }^{1-2, *}$ \\ ${ }^{1}$ FOTON, Systèmes Photoniques, UMR CNRS 6082, Université de Rennes 1, 22305 Lannion, France \\ ${ }^{2}$ ENIB, Technopôle Brest-Iroise, CS 73862, 29638 Brest, France
}

\begin{abstract}
The formal identification between a two-mode waveguide and a system of two mutually coupled single-mode waveguides stems from the symmetries of the evolution operator. When the gap tends to zero, the super-modes of the coupled system merge continuously into the modes of the multimode waveguide. For modelling purposes, it is very tempting to extend the analogy to three-mode waveguides (and beyond). But not without some precautions...
\end{abstract}

\section{Introduction}

Multimode waveguides are the core element for SpatialDivision Multiplexing (SDM), a technique where one wavelength can carry several channels of information, each one allocated to a specific modal distribution [1].

From the point of view of its effective indices, a twomode waveguide (TMWG) can be thought of as formally equivalent to a symmetric set of two coupled singlemode waveguides (SMWG) of wavevector $\beta$, with coupling constant $\chi$. When the gap tends towards zero, the even and odd super-modes of the coupled system, of wavevectors $\beta_{\text {even }}$ and $\beta_{\text {odd }}$, merge into the fundamental and first-order of the TMWG, respectively [2]. The identification proceeds as follows:

$$
\left\{\begin{array} { l } 
{ \beta _ { \text { even } } = \beta + \chi } \\
{ \beta _ { \text { odd } } = \beta - \chi }
\end{array} \Rightarrow \left\{\begin{array}{l}
\beta=\frac{\beta_{\text {even }}+\beta_{\text {odd }}}{2} \\
\chi=\frac{\beta_{\text {even }}-\beta_{\text {odd }}}{2}
\end{array}\right.\right.
$$

A similar extension of Coupled-Mode Theory (CMT) to a three-mode waveguide (3MWG) - and beyond - is tempting, but far from obvious. Investigating the ternary slab system as a reference case (whose properties can be calculated with arbitrary precision), we will observe that even in the basic symmetric configuration made of three identical SMWG, the simplest CMT model must be completed by at least one degree of freedom, in order for the propagation constants of the super-modes to be correctly accounted for.

In Section 2, we use a matrix formalism to derive the wavevectors and the transverse profile of the supermodes. In Section 3, we point out the discrepancies, before proposing several ways to recover the missing degree of freedom.

\section{The slab waveguide system}

The ternary slab system is depicted in Fig. 1:

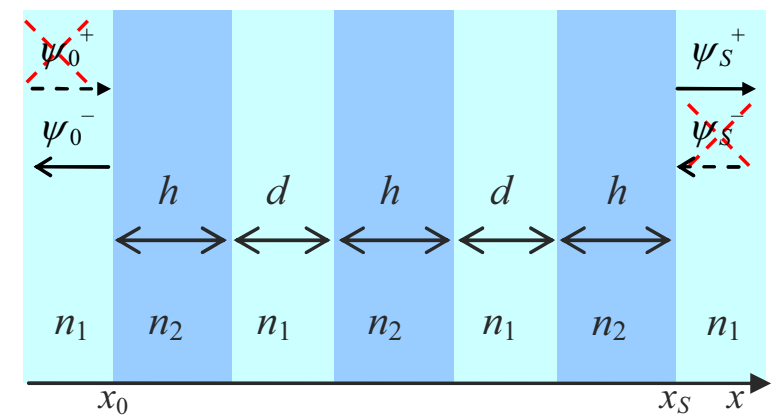

Fig. 1. The symmetric ternary slab system, made of three identical single-mode waveguides of higher index $n_{2}$ and width $h$, separated by a gap of lower index $n_{1}$ and width $d$. In the external cladding, only decaying evanescent waves are supported, imposing $\psi_{0}{ }^{+}=\psi_{S}^{-}=0$.

For numerical applications, wavelength in vacuum is $\lambda_{0}=1.5 \mu \mathrm{m} ; n_{1}=1 ; \quad n_{2}=1.5 ; \quad h=0,67 \mu \mathrm{m} ; k_{0}=\omega / c$. Time dependence is taken as $\mathrm{e}^{+i \omega t}$.

\subsection{Matrix description}

The matrix description of the slab system stems from the linearity of Maxwell equations [3-5]. For a given state of polarization (TE/TM), the transverse components of the wavevector of the isolated SMWG guided mode, of longitudinal propagation constant $\beta$, are either real $\left(\psi^{+}=A \mathrm{e}^{-i \kappa x}, \psi^{-}=B \mathrm{e}^{+i \kappa x}\right.$ if propagative $)$ or purely imaginary $\left(\psi^{+}=A \mathrm{e}^{-\gamma x}, \quad \psi^{-}=B \mathrm{e}^{+\gamma x}\right.$ if evanescent $)$, with $\quad \beta^{2}-\gamma^{2}=n_{1}^{2} k_{0}^{2}, \quad \beta^{2}+\kappa^{2}=n_{2}^{2} k_{0}^{2}>n_{1}^{2} k_{0}^{2}$. The boundary conditions ensure that no exponential increase is allowed in the semi-infinite cladding. 
The matrix of the isolated SMWG is such as:

$$
\begin{aligned}
& \left(\begin{array}{c}
0 \\
\psi_{0}^{-}
\end{array}\right)=\left(\begin{array}{ll}
m_{11} & m_{12} \\
m_{21} & m_{22}
\end{array}\right)\left(\begin{array}{c}
\psi_{S}^{+} \\
0
\end{array}\right), \\
& m_{11}=\cos \kappa h-\frac{1}{2}\left(\rho-\frac{1}{\rho}\right) \sin \kappa h, \\
& m_{12}=-\frac{1}{2}\left(\rho+\frac{1}{\rho}\right) \sin \kappa h=-m_{21}, \\
& m_{22}=\cos \kappa h+\frac{1}{2}\left(\rho-\frac{1}{\rho}\right) \sin \kappa h, \\
& \rho_{T E}=\frac{\mu_{1}}{\mu_{2}} \frac{\kappa h}{\gamma h}, \quad \rho_{T M}=\frac{\varepsilon_{1}}{\varepsilon_{2}} \frac{\kappa h}{\gamma h} .
\end{aligned}
$$

For the SMWG, the modal equation reads $m_{11}=0$, whereas for the $3 \mathrm{MWG}$, we get $M_{11}=0$, with:

$$
[\mathrm{M}]=[\mathrm{m}]\left(\begin{array}{cc}
\mathrm{e}^{\gamma d} & 0 \\
0 & \mathrm{e}^{-\gamma d}
\end{array}\right)[\mathrm{m}]\left(\begin{array}{cc}
\mathrm{e}^{\gamma d} & 0 \\
0 & \mathrm{e}^{-\gamma d}
\end{array}\right)[\mathrm{m}] .
$$

By solving the modal equation, as schematically depicted in Fig. 2, we obtain the quantized values of $\kappa h$; hence the values for the longitudinal wavevector $\beta$.

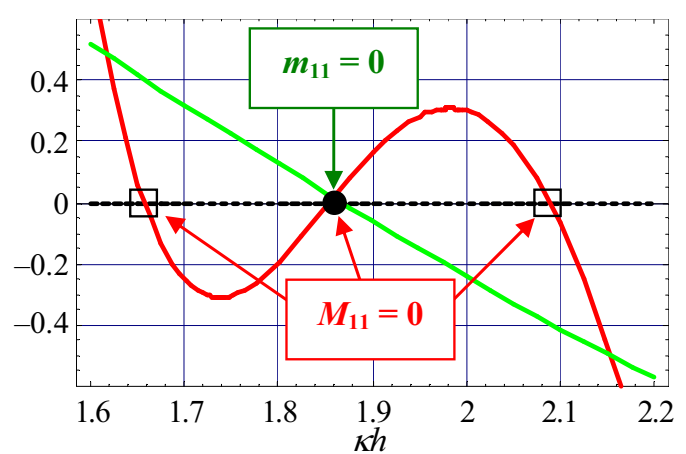

Fig. 2. Graphic depiction of the solution(s) for the TE modal equation, both for the SMWG $\left(m_{11}=0\right)$ and the $3 \mathrm{MWG}$ $\left(M_{11}=0\right)$, in the case where $d=h / 2$.

The three super-modes of the $3 \mathrm{MWG}$ are a slow (fundamental) one, a "neutral" one ( 1 st - order) and a fast one $\left(2^{\text {nd }}\right.$-order $)$, of respective wave-vectors $\left(\beta_{S}, \beta_{N}, \beta_{F}\right)$.

\subsection{Mode profile}

For several values of the gap $d$, we report in Fig. 3 the mode profile $\psi(x)=\psi^{+}+\psi^{-}$, normalized so that:

$$
\int|\psi(x)|^{2} d x=1 \text {. }
$$

For a vanishing gap $(d=0)$, the super-modes merge continuously into the fundamental, $1^{\text {st }}$-order and $2^{\text {nd }}$ order modes of a unique $3 \mathrm{MWG}$ of width $3 h$.
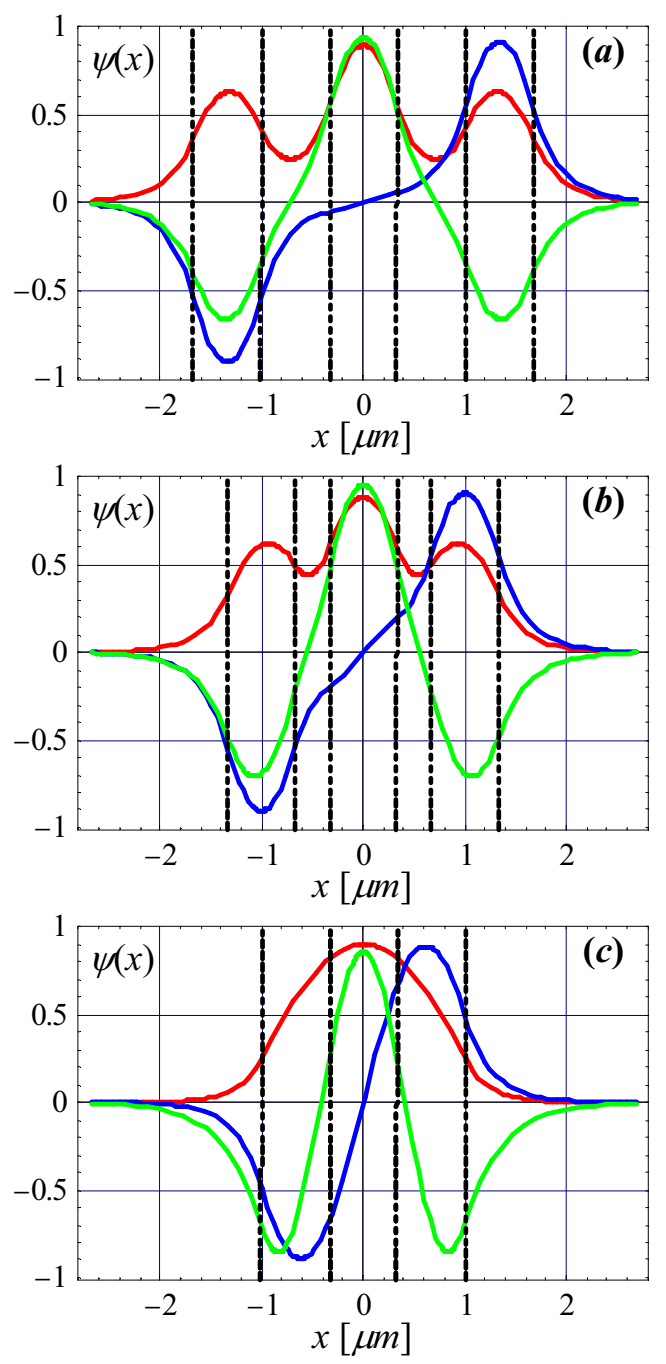

Fig. 3. TE mode profile (in complex amplitude) of the slow, "neutral" and fast super-modes of the ternary slab system: (a) $d=h$; (b) $d=h / 2$; (c) $d=0$.

\section{Coupled-Mode Theory}

\subsection{The simplest model}

The simplest model for the symmetric ternary slab system is based on intuitive, realistic assumptions: three identical SMWG, of propagation constant $\beta$, are similarly coupled to their nearest neighbour(s) through a real-valued coupling constant $\chi$. Instead of the three fields $\left(F_{1}, F_{2}, F_{3}\right)$, let us define the Slowly-Varying Envelope Amplitudes $A_{n}$ such as $F_{n}=A_{n} \mathrm{e}^{-i \beta z}$; these obey the evolution equation:

$$
i \frac{\partial}{\partial z}\left(\begin{array}{l}
A_{1} \\
A_{2} \\
A_{3}
\end{array}\right)=\chi\left(\begin{array}{lll}
0 & 1 & 0 \\
1 & 0 & 1 \\
0 & 1 & 0
\end{array}\right)\left(\begin{array}{l}
A_{1} \\
A_{2} \\
A_{3}
\end{array}\right) .
$$

By submitting the reduced operator to a standard diagonalization procedure, we easily derive the eigenvalue spectrum to find out that $\beta_{F}=\beta-\chi \sqrt{2}, \beta_{N}=\beta$, and $\beta_{S}=\beta+\chi \sqrt{2}$. 
Within this simplest model, the wavevectors of the three super-modes appear equidistant in $k$-space, which does not coincide with their actual distribution, as determined by an exact matrix analysis: (at least) one more degree of freedom is required.

\subsection{Corrective assumptions}

The first correction that comes to mind is a possible mismatch affecting the central waveguide, under the influence of its immediate surroundings [Fig. 4].

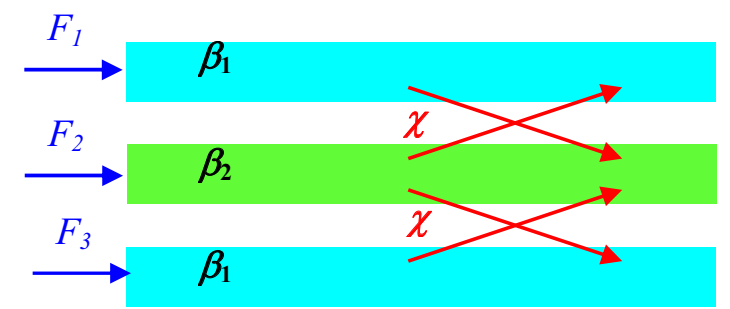

Fig. 4. Ternary slab system with central mismatch.

In this "diagonal perturbation" scheme, we have to consider three slightly different waveguides. The average wavevector $\beta$ and the mismatch $\Delta$ are:

$$
\beta=\frac{2 \beta_{1}+\beta_{2}}{3}, \quad \Delta=\frac{\beta_{2}-\beta_{1}}{3} .
$$

With $\delta=\Delta / \chi$, the new evolution equation is:

$$
i \frac{\partial}{\partial z}\left(\begin{array}{c}
A_{1} \\
A_{2} \\
A_{3}
\end{array}\right)=\chi\left(\begin{array}{ccc}
-\delta & 1 & 0 \\
1 & 2 \delta & 1 \\
0 & 1 & -\delta
\end{array}\right)\left(\begin{array}{c}
A_{1} \\
A_{2} \\
A_{3}
\end{array}\right) .
$$

The calculation of the mismatch-dependent eigenvalues is straightforward: with $R=8+9 \delta^{2}$, we get, for any $X \in\{F, N, S\}, \beta_{X}=\beta+\chi \lambda_{X}$, where

$$
\lambda_{F}=\frac{\delta-\sqrt{R}}{2}, \quad \lambda_{N}=-\delta, \quad \lambda_{S}=\frac{\delta+\sqrt{R}}{2} .
$$

Within this corrective model, the only knowledge of $\left(\beta_{F}, \beta_{N}, \beta_{S}\right)$ enables one to proceed to a complete identification of $(\beta, \chi, \Delta)$, since $\beta=\left(\beta_{F}+\beta_{N}+\beta_{S}\right) / 3$ and

$$
\chi=\sqrt{\frac{\left(\beta_{S}-\beta_{N}\right)\left(\beta_{N}-\beta_{S}\right)}{2}}, \quad \Delta=\beta-\beta_{N} .
$$

Unfortunately, another (fundamentally different) corrective assumption also ensures the missing degree of freedom: that of the "auxiliary coupling" [Fig. 5]:

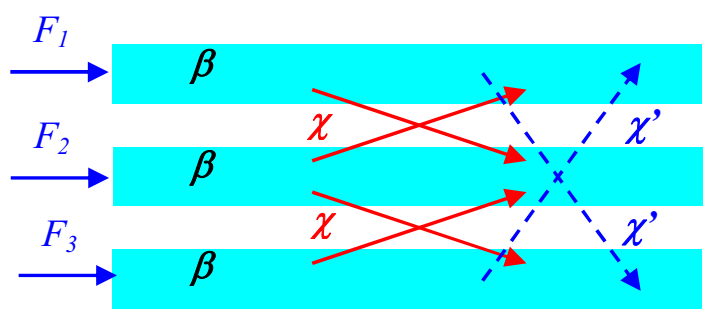

Fig. 5. Ternary phase-matched slab system with auxiliary coupling $\chi^{\prime}$ (with $\left|\chi^{\prime}\right|<<\chi$ ).
In this scheme, the waveguides are again identical, but there is an extra coupling $\chi$ ' between $F_{1}$ and $F_{3}$. With $\varepsilon=\chi^{\prime} / \chi$, the new evolution equation is:

$$
i \frac{\partial}{\partial z}\left(\begin{array}{l}
A_{1} \\
A_{2} \\
A_{3}
\end{array}\right)=\chi\left(\begin{array}{lll}
0 & 1 & \varepsilon \\
1 & 0 & 1 \\
\varepsilon & 1 & 0
\end{array}\right)\left(\begin{array}{l}
A_{1} \\
A_{2} \\
A_{3}
\end{array}\right) .
$$

The calculation of the coupling-dependent eigenvalues is straightforward: with $P=8+\varepsilon^{2}$, we get, for any $X \in\{F, N, S\}, \beta_{X}=\beta+\chi \lambda_{X}$, where

$$
\lambda_{F}=\frac{\delta-\sqrt{P}}{2}, \quad \lambda_{N}=-\delta, \quad \lambda_{S}=\frac{\delta+\sqrt{P}}{2} .
$$

Within this alternative corrective model, the only knowledge of $\left(\beta_{F}, \beta_{N}, \beta_{S}\right)$ enables one - once again - to proceed to a complete identification of $(\beta, \chi, \Delta)$, since $\beta=\left(\beta_{F}+\beta_{N}+\beta_{S}\right) / 3, \Delta=\beta-\beta_{N}$, and

$$
\chi=\sqrt{\frac{\left(2 \beta_{S}-\beta_{N}-\beta_{F}\right)\left(\beta_{S}+\beta_{N}-2 \beta_{F}\right)}{18}} .
$$

But there is no obvious reason to choose between the two possible corrections: the modal equation doesn't provide enough information to settle the matter.

\section{Conclusions}

In the frame of Coupled-Mode Theory, we can exploit the properties of the super-modes in order to derive the formal identity between a 3-mode waveguide and a set of three mutually coupled single-mode waveguides. On the "textbook" example of slab systems, we show that the simplest model for a ternary coupler, although based on intuitive realistic assumptions, is incomplete. We explore two different corrective hypotheses that lead to similar results in terms of mode indices. This means that, if a strict term-to-term equivalence is actually legitimate, the solution is not unique. We are currently investigating another criterion: the super-mode profile.

This work is supported by the Labex CominLabs (French National Research Agency program "Investing for the Future" ANR-10-LABX-07-01) within the "3D-Optical-ManyCores" Project (http://www.3d-opt-many-cores.cominlabs.ueb.eu/). The author would also like to thank Prof. Patrice Féron and Dr. Alberto Parini (FOTON Sys.Phot.) for helpful discussions.

\section{References}

1. D. Dai, J.E. Bowers, Nanophotonics, 3, 4-5, 283311 (2014)

2. Y.G. Boucher, Opt. Eng., 53,7, 071810 (2014)

3. A. Yariv, P. Yeh, Optical Waves in Crystals (Wiley, New York, 1984)

4. T. Tamir (Ed.), Guided-Wave Optoelectronics (Springer-Verlag, Berlin, 1990)

5. C.-L. Chen, Foundations for Guided-Wave Optics (Wiley, Hoboken, 2007) 